\title{
Atacama Compact Array observations of the pulsar-wind nebula of SNR 0540-69.3
}

\author{
P. Lundqvist ${ }^{\oplus},{ }^{1,2 \star}$ N. Lundqvist,${ }^{1}$ C. Vlahakis,${ }^{3}$ C.-I. Björnsson, ${ }^{1}$ J. R. Dickel, ${ }^{4}$ \\ M. Matsuura ${ }^{\odot},{ }^{5}$ Yu. A. Shibanov, ${ }^{6,7}$ D. A. Zyuzin ${ }^{\oplus 6}$ and G. Olofsson ${ }^{1}$ \\ ${ }^{1}$ Department of Astronomy, AlbaNova University Center, Stockholm University, SE-10691 Stockholm, Sweden \\ ${ }^{2}$ The Oskar Klein Centre, AlbaNova, SE-10691 Stockholm, Sweden \\ ${ }^{3}$ National Radio Astronomy Observatory, 520 Edgemont Road, Charlottesville, VA 22903-2475, USA \\ ${ }^{4}$ Department of Astronomy, University of Illinois Urbana-Champaign, 1002 W. Green Street, Urbana, IL 61801, USA \\ ${ }^{5}$ School of Physics and Astrophysics, Cardiff University, Queens Buildings, The Parade, Cardiff CF24 3AA, UK \\ ${ }^{6}$ Ioffe Institute, Politekhnicheskaya 26, St Petersburg 194021, Russia \\ ${ }^{7}$ Peter the Great St. Petersburg Polytechnic University, Politekhnicheskaya 29, St Petersburg 195251, Russia
}

Accepted 2020 June 6. Received 2020 May 5; in original form 2019 June 16

\begin{abstract}
We present observations of the pulsar-wind nebula (PWN) region of SNR 0540-69.3. The observations were made with the Atacama Compact Array (ACA) in Bands 4 and 6. We also add radio observations from the Australia Compact Array at $3 \mathrm{~cm}$. For $1.449-233.50 \mathrm{GHz}$, we obtain a synchrotron spectrum $F_{v} \propto v^{-\alpha_{v}}$, with the spectral index $\alpha_{v}=0.17 \pm 0.02$. To conclude how this joins the synchrotron spectrum at higher frequencies, we include hitherto unpublished AKARI mid-infrared data, and evaluate published data in the ultraviolet (UV), optical, and infrared (IR). In particular, some broad-band filter data in the optical must be discarded from our analysis due to contamination by spectral line emission. For the UV/IR part of the synchrotron spectrum, we arrive at $\alpha_{v}=0.87_{-0.10}^{+0.08}$. There is room for $2.5 \times 10^{-3} \mathrm{M}_{\odot}$ of dust with a temperature of $\sim 55 \mathrm{~K}$ if there are dual breaks in the synchrotron spectrum, one around $\sim 9 \times 10^{10} \mathrm{~Hz}$ and another at $\sim 2 \times 10^{13} \mathrm{~Hz}$. The spectral index then changes at $\sim 9 \times 10^{10} \mathrm{~Hz}$ from $\alpha_{v}=0.14 \pm 0.07$ in the radio to $\alpha_{v}=0.35_{+0.05}^{-0.07}$ in the millimetre-to-far-IR range. The ACA Band 6 data marginally resolve the PWN. In particular, the strong emission $\sim 1^{\prime \prime} 5$ south-west of the pulsar, seen at other wavelengths, and resolved in the $3 \mathrm{~cm}$ data with its $0 . " 8$ spatial resolution, is also strong in the millimetre range. The ACA data clearly reveal the supernova remnant shell $\sim 20-35$ arcsec west of the pulsar, and for the shell we derive $\alpha_{v}=0.64 \pm 0.05$ for the range 8.6-145 GHz.
\end{abstract}

Key words: pulsars: individual: PSR B0540-69.3 - supernovae: general-ISM: SNR 054069.3 - ISM: supernova remnants - Magellanic Clouds.

\section{INTRODUCTION}

The oxygen-rich ejecta of the $\sim 10^{3}$ yr old Large Magellanic Cloud (LMC) supernova remnant (SNR) 0540-69.3 (in short SNR0540; Kirshner et al. 1989; Serafimovich et al. 2005) are evidence that SNR0540 is the result of an explosion of a massive, $\sim 20 \mathrm{M}_{\odot}$ (at zero-age main-sequence), progenitor (Chevalier 2006; Williams et al. 2008; Lundqvist et al. 2011). The radiation emitted from the remnant manifests itself mainly through radio and $\mathrm{X}$-ray emission from a shell of radius $\sim 20-35$ arcsec, corresponding to $\sim 4.9-8.5 \mathrm{pc}$ at the $50 \mathrm{kpc}$ distance of LMC (Manchester et al. 1993b; Gotthelf \&

^E-mail: peter@astro.su.se
Wang 2000). Most of this emission comes from the western part of the shell.

The remnant contains the pulsar PSR B0540-69.3 (henceforth PSR0540), discovered as a pulsed $(P=50.2 \mathrm{~ms}) \mathrm{X}$-ray source by Seward et al. (1984). Pulsations have subsequently also been detected in the optical (Middleditch \& Pennypacker 1985), at radio wavelengths (Manchester et al. 1993a), and recently in the ultraviolet (UV; Mignani et al. 2019). The properties of PSR0540 are very similar to those of the Crab pulsar: it spins rapidly and it is young (spin-down age $1660 \mathrm{yr}$ ).

Like the Crab pulsar, PSR0540 powers a pulsar-wind nebula (PWN), which we will refer to as PWN0540. The full size of PWN0540 in radio is $\sim 7-8$ arcsec across, with an inner core of $\sim 5$ arcsec in diameter, also seen in the UV/optical (Caraveo et al. 
1992; Serafimovich et al. 2004) radio (e.g. Manchester et al. 1993b) and X-rays (Gotthelf \& Wang 2000). The PWN emits synchrotron emission with a flux $F_{v} \propto v^{-\alpha_{v}}$, where $\alpha_{v}$ runs from $\sim 0.15$ in the radio (Brantseg et al. 2014) to a larger value in the optical (Serafimovich et al. 2004; Mignani et al. 2012).

PWN0540 is in no sense different from other PWNe when it comes to the shallow slope in radio; the vast majority of $39 \mathrm{PWNe}$ studied in the radio have $\alpha_{v} \lesssim 0.3$ (Reynolds et al. 2017). Neither is the steepening towards higher photon energies unique. For example, the Crab PWN has a spectral break around $\sim 3 \times 10^{10} \mathrm{~Hz}$, where the spectral index changes from $\alpha_{v} \approx 0.3$ in the radio to $\alpha_{v} \approx 0.42$ at higher frequencies (Gomez et al. 2012). Such spectral breaks have also been seen in other PWNe (cf. Reynolds et al. 2017, and references therein).

The shape of the synchrotron radio/infrared (IR) spectrum provides important information about the energy distribution of relativistic electrons and their radiative losses, and inhomogeneities within the PWN (Reynolds et al. 2017). For a power-law distribution for the energy of relativistic electrons, $\mathrm{d} N / \mathrm{d} E=N_{0} E^{-p}$, where $E=$ $\gamma m_{\mathrm{e}} c^{2}$ is the energy of the electrons and $\gamma$ is the Lorentz factor, the intensity of optically thin synchrotron emission is $\alpha v^{-\alpha}$, where $\alpha=(p-1) / 2$. A flat spectrum with $\alpha \sim 0.15$, as for PWN0540 in the radio, would then indicate $p \sim 1.3$. This is much shallower than $p \gtrsim 2$ expected at shock fronts of relativistic shocks (e.g. Bykov et al. 2012), corresponding to $\alpha \gtrsim 0.5$. Bucciantini et al. (2011) showed that a broken power-law energy distribution of the relativistic electrons can explain the shallow radio spectrum of the Crab PWN and a few other PWNe, and argued for that the relativistic electrons at the lowest energies could be accelerated in a turbulence zone in connection with the termination shock rather than via Fermi acceleration. In the work of Bucciantini et al. (2011), as well of others (e.g. Gelfand et al. 2009; Martin et al. 2012), particles are continuously injected into the PWN, with the energy spectrum of the injected particles being discontinuous or having a break.

There are models invoking reconnection as the source of particle acceleration (e.g. Sironi \& Spitkovsky 2011; Cerutti et al. 2012; Clausen-Brown \& Lyutikov 2012; Porth et al. 2016), and Lyutikov et al. (2019) argue that both Fermi-I and reconnection processes occur in the Crab PWN, and that this can explain the multiple spectral breaks for that PWN. Several spectral breaks are also evident in compiled IR-optical-X-ray spectra of the torus-like parts of other well-studied PWNe (PWN0540, 3C 58, J1124-5916, and G21.5-0.9; Zharikov et al. 2013), implying that multiple populations of relativistic particles are responsible for the emission in different spectral domains. In particular, the overall radio-to-X-ray spectrum of PWN0540 has been discussed by Serafimovich et al. (2004), Mignani et al. (2012), Lundqvist et al. (2011), and Brantseg et al. (2014), and it shows that the X-ray emission is stronger than expected from a power-law extrapolation of the IR/optical/UV spectrum. This indeed indicates the existence of more than one emission component. In addition, spatially resolved X-ray spectra of PWN0540 show that the steepness of the X-ray spectrum increases away from the torus region (Lundqvist et al. 2011), which is expected if the particles injected there experience adiabatic and synchrotron losses as they move away from that region. Such spatial variations tend to smooth spectral breaks in spatially integrated spectra of PWNe (e.g. Reynolds et al. 2017).

An established synchrotron contribution in the millimetre/IR range is needed to estimate possible additional continuum emission from supernova-produced dust. Prime examples of the latter in SNRs are the Crab Nebula (Gomez et al. 2012; Owen \& Barlow 2015), PWN G54.1+0.3 (Temim et al. 2017; Rho et al. 2018), Cas
Table 1. ACA and AKARI observations of SNR 0540-69.3.

\begin{tabular}{lccc}
\hline $\begin{array}{l}\text { Time } \\
\text { UT }\end{array}$ & Band & $\begin{array}{c}\text { Integration } \\
\text { s }\end{array}$ & $\begin{array}{c}\text { Frequency } \\
\text { GHz }\end{array}$ \\
\hline 2018 March 19.96 & ACA/Band 4 & 438.48 & $138^{a}$ \\
& & & $140^{a}$ \\
& & & $150^{a}$ \\
& & & $152^{a}$ \\
2018 March 25.94 & ACA/Band 6 & 665.28 & $214.5^{a}$ \\
& & & $216.5^{a}$ \\
& & & $230.5^{a}$ \\
2006 October 31.83 & AKARI/L24 & $148.75^{b}$ & $(1.044-1.629) \times 10^{4}$ \\
2006 October 31.21 & AKARI/L15 & $148.75^{b}$ & $(1.396-2.456) \times 10^{4}$ \\
2006 October 25.98 & AKARI/S11 & $148.75^{b}$ & $(1.960-3.625) \times 10^{4}$ \\
2006 October 25.97 & AKARI/S7 & $148.75^{b}$ & $(3.429-5.423) \times 10^{4}$ \\
2006 October 25.98 & AKARI/N3 & $147.0^{b}$ & $(7.519-11.82) \times 10^{4}$
\end{tabular}

Notes. ${ }^{a}$ Average frequency of each spectral window (spw). The spw bandwidth is $1.875 \mathrm{GHz}$.

${ }^{b}$ Net integration times including both long and short exposures in three dithered sky positions.

Table 2. ACA continuum parameters for SNR 0540-69.3.

\begin{tabular}{lcccc}
\hline Band & $\begin{array}{c}\text { Frequency } \\
\mathrm{GHz}\end{array}$ & $\begin{array}{c}S_{v, \text { peak }}^{a} \\
\text { mJy beam }^{-1}\end{array}$ & $\begin{array}{c}\sigma_{\text {rms }} \\
\mathrm{mJy}\end{array}$ & $\begin{array}{c}\theta_{\text {beam }}^{b} \\
\text { size (PA) }\end{array}$ \\
\hline 4 & 145 & $30.97 \pm 1.58$ & 0.291 & $10^{\prime \prime} .79 \times 8^{\prime \prime} .33(84.09)$ \\
6 & 223.5 & $19.33 \pm 1.96$ & 0.348 & $7^{\prime \prime} .52 \times 5^{\prime \prime} .18(-83.60)$ \\
\hline
\end{tabular}

Notes. ${ }^{a}$ Peak flux density. Uncertainties are the rms noise level given in column 4 , combined with a 5 per cent absolute flux calibration uncertainty for Band 4, and a 10 per cent uncertainty for Band 6 (cf. Section 3.2).

${ }^{b}$ Synthesized beam size (and beam position angle; see Section 2.2).

A (De Looze et al. 2017), and the young ( $\lesssim 2.5 \mathrm{kyr}$ ) remnants G11.2-0.3, G21.5-0.9, and G29.7-0.3 (Chawner et al. 2019). The most obvious case outside the Milky Way is SN 1987A (Matsuura et al. 2011, 2015; Indebetouw et al. 2014; Dwek \& Arendt 2015), which, like the SNRs mentioned, has substantial amounts of dust ( $\gtrsim 0.2 \mathrm{M}_{\odot}$ ) embedded within it.

Spitzer observations indicate that dust also exists in PWN0540. The estimated temperature and mass of this dust are $\sim 50-65 \mathrm{~K}$ and (1-3) $\times 10^{-3} \mathrm{M}_{\odot}$, respectively (Williams et al. 2008). These estimates are, however, sensitive to the shape of the underlying synchrotron spectrum in the IR. Williams et al. (2008) used the results of Serafimovich et al. (2004) in the UV and optical to extrapolate to the IR. A critical assessment of the UV/optical synchrotron component is therefore essential to derive a reliable dust mass. The more recent estimate by Mignani et al. (2012) for the UV/optical/near-IR synchrotron spectrum indicates a notably higher flux than that of Serafimovich et al. (2004), and the derived synchrotron slope is markedly shallower. If the results of Mignani et al. (2012) were to be combined with the Spitzer data, this would seriously affect the derived mass and temperature of the dust discussed by Williams et al. (2008).

To better constrain the continuum emission from PWN0540, we have obtained data from the Atacama Compact Array (ACA) in Bands 4 and 6, i.e. in the frequency range $137.0-233.5 \mathrm{GHz}^{1}$ (cf. Tables 1 and 2). We have also complemented the millimetre ACA data with the high-resolution radio data of Dickel et al. (2002) to model the partially resolved ACA data. The reason for this modelling is the uneven spatial distribution of emission

${ }^{1}$ ALMA Program 2017.1.01391.S, PI: P. Lundqvist. 
Table 3. Spatial resolution of the ACA, AKARI, and ATCA data for SNR 0540-69.3.

\begin{tabular}{lccc}
\hline Instrument & Band & $\begin{array}{c}\text { Frequency } \\
\text { GHz }\end{array}$ & Beam size \\
\hline ATCA & $3 \mathrm{~cm}$ & 8.64 & $0^{\prime \prime} 8^{a}$ \\
ACA & Band 4 & 145 & $10^{\prime} \cdot 79 \times 8^{\prime \prime} .33^{b}$ \\
ACA & Band 6 & 223.5 & $7^{\prime \prime} .52 \times 5^{\prime \prime} .18^{b}$ \\
AKARI & MIR-L L24 & $1.30 \times 10^{4}$ & $6^{\prime \prime} 8^{c}$ \\
AKARI & MIR-L L15 & $1.92 \times 10^{4}$ & $5^{\prime \prime} 7^{c}$ \\
AKARI & MIR-S S11 & $2.84 \times 10^{4}$ & $4^{\prime \prime} 8^{c}$ \\
AKARI & MIR-S S7 & $4.16 \times 10^{4}$ & $5^{\prime \prime} 1^{c}$ \\
AKARI & NIR N3 & $9.55 \times 10^{4}$ & $4^{\prime \prime} 0^{c}$ \\
\hline
\end{tabular}

Notes. ${ }^{a}$ Synthesized beam size (cf. Dickel et al. 2002).

${ }^{\mathrm{b}}$ Synthesized beam size (cf. Table 2).

${ }^{\mathrm{c}} \mathrm{FWHM}$ according to Onaka et al. (2007).

seen in the PWN at many wavelengths. In particular, much of the emission, especially in X-rays, but also in the optical, comes from a region $\sim 1^{\prime \prime} .5$ south-west of the pulsar, where a bright time-variable structure ('blob') can be seen (De Luca et al. 2007; Lundqvist et al. 2011). This is also the region where some of the highest densities, $N_{\mathrm{e}} \sim 2 \times 10^{3} \mathrm{~cm}^{-3}$, in the PWN can be found (Sandin et al. 2013). In addition to the ACA and Australia Compact Array (ATCA) data, we have also used IR data collected by the AKARI satellite in 2006 for the wavelength range 2.53-28.7 $\mu \mathrm{m}$, to add to the Spitzer data.

The paper is organized as follows: In Section 2, we describe and discuss the ATCA, ACA, and AKARI observations, and in Section 3 we analyse the observations. In Section 4, we put the results into context, and in Section 5 we summarize our conclusions.

\section{OBSERVATIONS}

\subsection{ATCA observations}

SNR 0540-69.3 has been observed with ATCA at several frequencies on several occasions. We have used the $3 \mathrm{~cm}$ data first presented in Dickel et al. (2002) and subsequently in Brantseg et al. (2014, cf. their table 1). These data were obtained on 1995 October 23-24 UT under ATCA Program C014. Polarization was registered (cf. Dickel et al. 2002), but is not utilized here. The data were reduced using MIRIAD version $1.0^{2}$ (Sault et al. 1995), setting robust $=0$, and the cell size to 0 '.25 to get adequate oversampling to reveal features with a size of $\lesssim 1 \mathrm{arcsec}$. The image was then cleaned using MIRIAD CLEAN version 1.0 with gain $=0.02$ and niter $=200000$. Finally, restoration was done with MIRIAD RESTOR version 1.2 setting full width at half-maximum $(\mathrm{FWHM})=1.0$, i.e. a circularly symmetric Gaussian beam was assumed with an FWHM of 1".0. The final image had a spatial resolution with a half-power beam-width (HPBW) of $0 . " 8$ (cf. Table 3). The spatial resolution is thus superior to the $3 \mathrm{~cm}$ image discussed by Brantseg et al. (2014), which has a beam size of $1^{\prime \prime} .9 \times 1^{\prime \prime} .5$. We have used our image for the analysis here, except for the integrated flux density from the PWN at $3 \mathrm{~cm}$, which we took from Brantseg et al. (2014), as they used natural weighting for the robustness that trades spatial resolution for slightly better signal-to-noise.

As discussed by Serafimovich et al. (2005) and Lundqvist et al. (2011), the positional uncertainty of the pulsar, and thus the PWN, is larger in the radio $(\sim 2 \operatorname{arcsec})$ and in X-rays $\left(\sim 0^{\prime \prime} .5\right)$ than in the
UV/optical ( $\lesssim 0{ }^{\prime}$. 1; Mignani et al. 2019). The alignment between optical and X-ray images was discussed in Lundqvist et al. (2011), and is used in Fig. 3. Based on the arguments in Section 3.1, we have aligned the ATCA data with the optical data in a similar way. As our analysis focuses on the PWN with its $\sim 7-8$ arcsec extent, positional uncertainty is not a source of overall uncertainty for our analysis.

\subsection{ACA observations}

We observed the millimetre/submillimetre emission in SNR 054069.3 using ACA in Band 4 (145 GHz) and Band 6 (223.5 GHz) as part of Atacama Large Millimeter/submillimeter Array (ALMA) programme 2017.1.01391.S. The data were obtained on 2018 March 19 and 25 for Bands 4 and 6, respectively, and the array consisted of 10-11 7-m antennas. A single pointing was observed in each band. The total on-source integration times were 438.5 and $665.3 \mathrm{~s}$, in Bands 4 and 6, respectively. The spectral set-up covered a total usable bandwidth of $7.5 \mathrm{GHz}$, consisting of four $2 \mathrm{GHz}(1.875 \mathrm{GHz}$ usable), 128 channel spectral windows (spws) and dual polarization. The four spws were centred at 138, 140, 150, and $152 \mathrm{GHz}$ for Band 4 and 214.5, 216.5, 230.5, and 232.5 GHz for Band 6. The observing parameters are summarized in Table 1.

The data were reduced using the Common Astronomy Software Applications (CASA) package (McMulllin et al. 2007). We used the calibrated uv data delivered by ALMA, which was calibrated using the ALMA Pipeline, but we performed our own imaging to improve signal-to-noise and image quality (the delivered image products were undercleaned). The calibrated Band 4 and Band 6 data sets, respectively, contained data from 10 and 9 antennas. We carried out imaging of the continuum emission, utilizing the total $7.5 \mathrm{GHz}$ bandwidth, using the CASA task TCLEAN with Briggs weighting and a robust $=0.5$ weighting of the visibilities, using CASA version 5.4.0. We also performed self-calibration. For the Band 4 data, we performed several rounds of phase-only self-calibration, initially with a $300 \mathrm{~s}$ time interval followed by refinement with shorter time intervals down to a $30 \mathrm{~s}$ interval. Amplitude self-calibration was then performed with a time interval of $30 \mathrm{~s}$. For the Band 6 data, we performed one round each of phase-only and amplitude selfcalibration, with a time interval of $300 \mathrm{~s}$. The resulting images are shown in Fig. 1, and the resulting synthesized beam sizes and rms noise levels are given in Table 2. Self-calibration produced a factor of about 2 improvement in the $\mathrm{S} / \mathrm{N}$ for the Band 4 data compared to deeper cleaning alone (and a factor of several compared to the ALMA-delivered shallow-cleaned images). For Band 6, selfcalibration only produced a slight improvement (at the 20 per cent level) in the $\mathrm{S} / \mathrm{N}$ compared to our deeper cleaning alone, but the appearance of imaging artefacts was improved.

\subsection{AKARI observations}

SNR 0540-69.3 was observed in 2006 by AKARI as part of an AKARI IR survey of the Magellanic clouds (Kato et al. 2012). Five bands covered the range 2.53-28.7 $\mu \mathrm{m}$, which overlaps with the wavelength range of previously reported Spitzer observations (Williams et al. 2008). Processed data were retrieved from the AKARI-LMC Point Source Catalogue, ${ }^{3}$ and entries for PWN0540 were identified for all imaging filters in the catalogue, i.e. NIR 

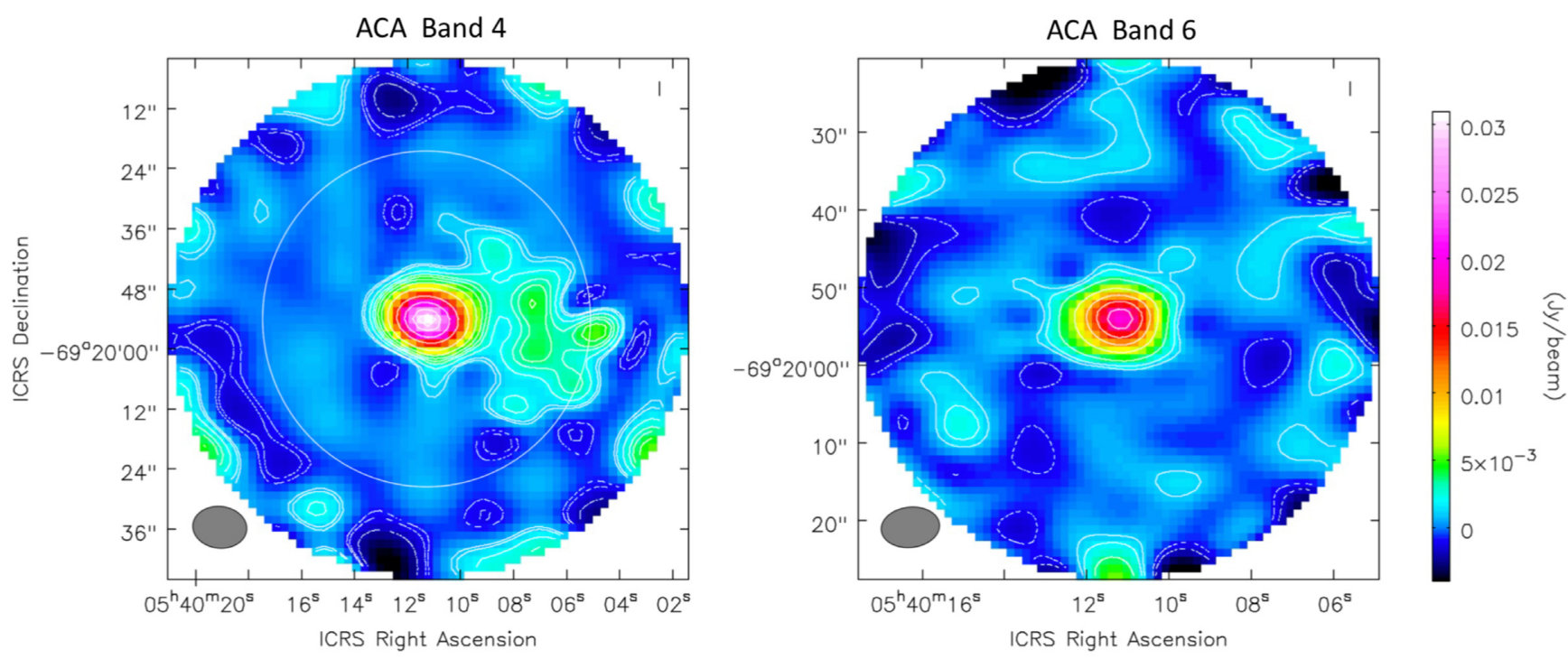

Figure 1. ACA Band 4 (left-hand panel) and Band 6 (right-hand panel) images of SNR 0540-69.3. The rms noise levels are 0.291 and $0.348 \mathrm{mJy}$, for Bands 4 and 6, respectively. The signal-to-noise (peak flux density divided by rms) for the central part of the PWN in Band 4 is $\approx 106$, and for Band 6 it is $\approx 56$. For Band 4 , the western part of the remnant shell at $20-35$ arcsec from the centre is clearly visible. Structures at the edges of the maps are not real. The faintest intensity shown by solid white contours marks the $3 \sigma$ intensity level. The Band 4 contours shown are at $-7 \sigma,-4 \sigma,-3 \sigma, 3 \sigma, 4 \sigma, 7 \sigma, 10 \sigma, 12 \sigma, 15 \sigma, 20 \sigma, 50 \sigma, 70 \sigma$, $90 \sigma$, and $100 \sigma$, and the Band 6 contours are at $-5 \sigma,-3 \sigma, 3 \sigma, 5 \sigma, 10 \sigma, 15 \sigma, 20 \sigma, 30 \sigma, 40 \sigma$, and $50 \sigma$. The ACA Band 4 beam has size $10^{\prime \prime} .79 \times 8.33$ and orientation PA $=84 .{ }^{\circ} 09$, and the corresponding numbers for Band 6 are $77^{\prime \prime} 52 \times 5^{\prime \prime} .18$ and PA $=-83 .{ }^{\circ} 60$. The beams are displayed in the lower left corners of each panel. The intensity scale is the same for both the panels. For a comparison, the Band 6 field of view is highlighted as a white circle in the Band 4 image.

N3 $(3.2 \mu \mathrm{m})$, MIR-S/S7 $(7.0 \mu \mathrm{m})$, MIR-S/S11 $(11 \mu \mathrm{m})$, MIRL/L15 $(15 \mu \mathrm{m})$, and MIR-L/L24 $(24 \mu \mathrm{m})$. The observations are summarized in Table 1, and the spatial resolution in the various filter is listed in Table 3. The pixel scales in NIR, MIR-S, and MIR-L images are 1."446, 2."340, and 2."384, respectively. The photometric values in the catalogue were obtained using a radius of 10 pixels for $\mathrm{N} 3$, and 3 pixels for other band images. PWN0540 was therefore fully covered by the aperture photometry, although marginally so for the MIR-L/L24 band.

\section{RESULTS}

\subsection{Morphology revealed by ACA and ATCA}

Fig. 1 displays the ACA Band 4 and 6 data. The beams and their orientations are highlighted as HPBW ovals in the lower left corners of the panels, and are described in the figure caption. The central PWN is clearly seen in both bands, as well as the SNR emission to the west out to $\sim 35$ arcsec for Band 4 . Band 6 with its smaller field of view and lower signal-to-noise cannot reliably trace the remnant shell (see also Fig. 2).

A $70 \operatorname{arcsec} \times 70 \operatorname{arcsec} 3 \mathrm{~cm}$ map of SNR 0540-69.3, using the data discussed in Section 2.1, is shown in the left-hand panel of Fig. 2. Apart from the well-known strong emission on the west side, $\sim 20-35$ arcsec from the pulsar, there is weaker emission in a seemingly east-west symmetry. In general, the outer remnant emission is more extended on the southern side, reaching in south-eastern direction as far as it does to the south-west. The extended structure on the south-eastern side has not been revealed in previously published radio images, although emission in this region has been displayed in X-rays (Park et al. 2010). A fuller comparison between X-rays and radio for the SNR shell is made in Brantseg et al. (2014). The middle and right-hand panels of Fig. 2 show ACA Band 4 and Band 6 maps, respectively, with the $3 \mathrm{~cm}$ data being overlaid. The larger HPBW of the ACA data smears out the remnant structures revealed by ATCA, but there is a clear overlap of the remnant shell between radio and ACA Band 4. There is also a hint of overlap between radio and Band 6, but a deeper image is needed to draw firm conclusions.

Closing in on the PWN, both the ACA bands have beam sizes larger (Band 4), or of roughly the same size (Band 6) as the PWN. No obvious structures of the PWN are revealed in the ACA bands. This is contrasted by the detailed structure of the PWN depicted in the $3 \mathrm{~cm}$ radio image in the middle panel of Fig. 3, where the radio image is drawn together with X-ray and optical images on the same scale. The X-ray and optical images are from our previous study in Lundqvist et al. (2011). The X-ray data were taken with Chandra HRC-I in 2000 June in the $0.2-10 \mathrm{keV}$ band, and the optical HST/F547M continuum data are from1999 and 2005, The optical data shown in Fig. 3 were wavelet filtered by Lundqvist et al. (2011) to more clearly bring out structures of the PWN.

The 0." 8 HPBW of the radio data is shown in Fig. 3 as a filled red circle. The spatial resolution is good enough for the $3 \mathrm{~cm}$ emission to clearly reveal the two strongest continuum sources, namely the pulsar and its immediate surroundings, as well as the blob $\sim 1$ 1.5 south-west of the pulsar. The two centres of emission were revealed in radio for first time by Dickel et al. (2002), but there it was suggested that the pulsar was the object to the south-west. A comparison between the three panels of Fig. 3 clearly shows that this is not the case, and to guide the eye, we have drawn grey horizontal lines in the east-west direction through the position of the pulsar and through the blob at its 1999 position. As discussed by De Luca et al. (2007) and Lundqvist et al. (2011), the emission centre of the blob in the optical appears to move over time towards the south-west. This is visible in the panel to the right of Fig. 3 where the 1999 structure in the optical is overlaid on that from 


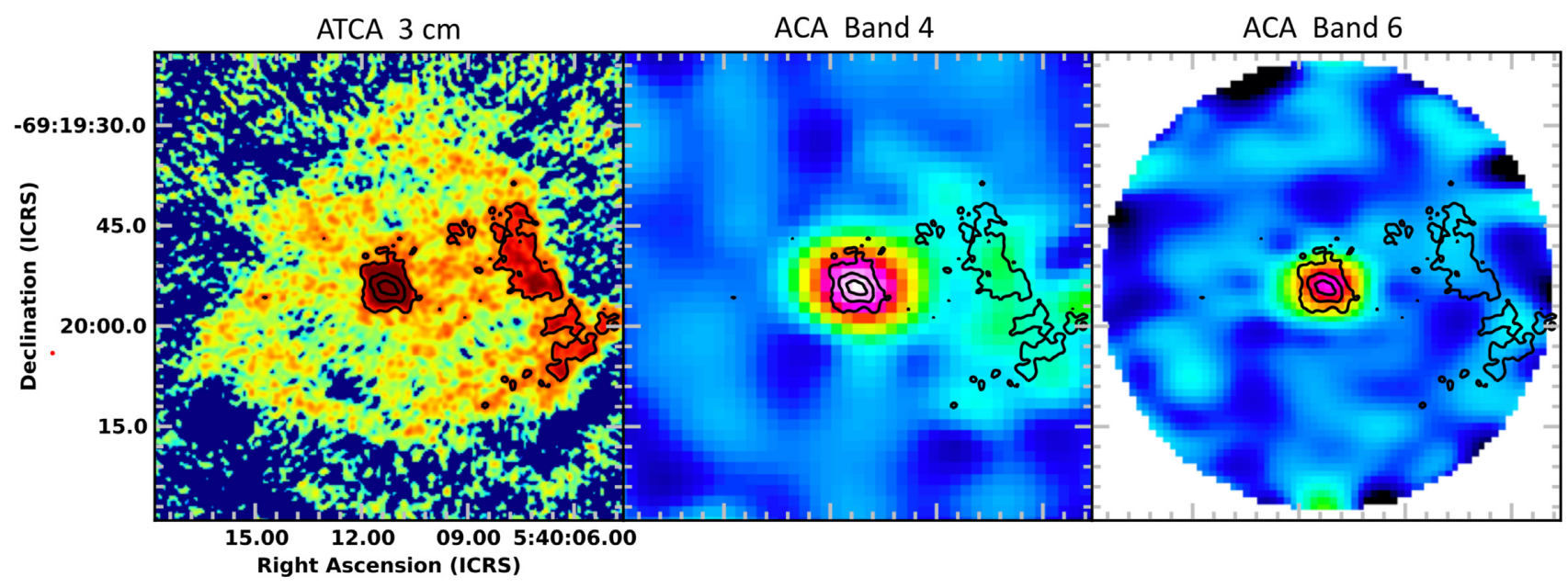

Figure 2. 70 arcsec $\times 70$ arcsec maps of PWN0540 in radio ( $3 \mathrm{~cm}$, ATCA), and at millimetre wavelengths ( 2 and $1.3 \mathrm{~mm}$, ALMA/ACA Bands 4 and 6; middle and right-hand panels, respectively), covering the PWN and remnant shell. The axes in all the three panels are the same. For a comparison, and to aid the eye, contours showing the $3 \mathrm{~cm}$ emission at levels of $0.2,1.0$, and $2.0 \mathrm{mJy}_{\text {beam }}^{-1}$ are overlaid on all the maps. In the $3 \mathrm{~cm}$ map the HPBW is $0 .{ }^{\prime \prime} 8$ (Dickel et al. 2002), and the PWN size is $\sim 8 \operatorname{arcsec} \times 7$ arcsec, so the PWN is fully resolved. Details for the ACA maps, including the beam sizes and intensity scale, are the same as in Fig. 1. With the larger beams in the millimetre maps, the PWN is only partially resolved (see the discussion in the text). Note the similar structures of the western part of the remnant shell in radio and in ACA Band 4.

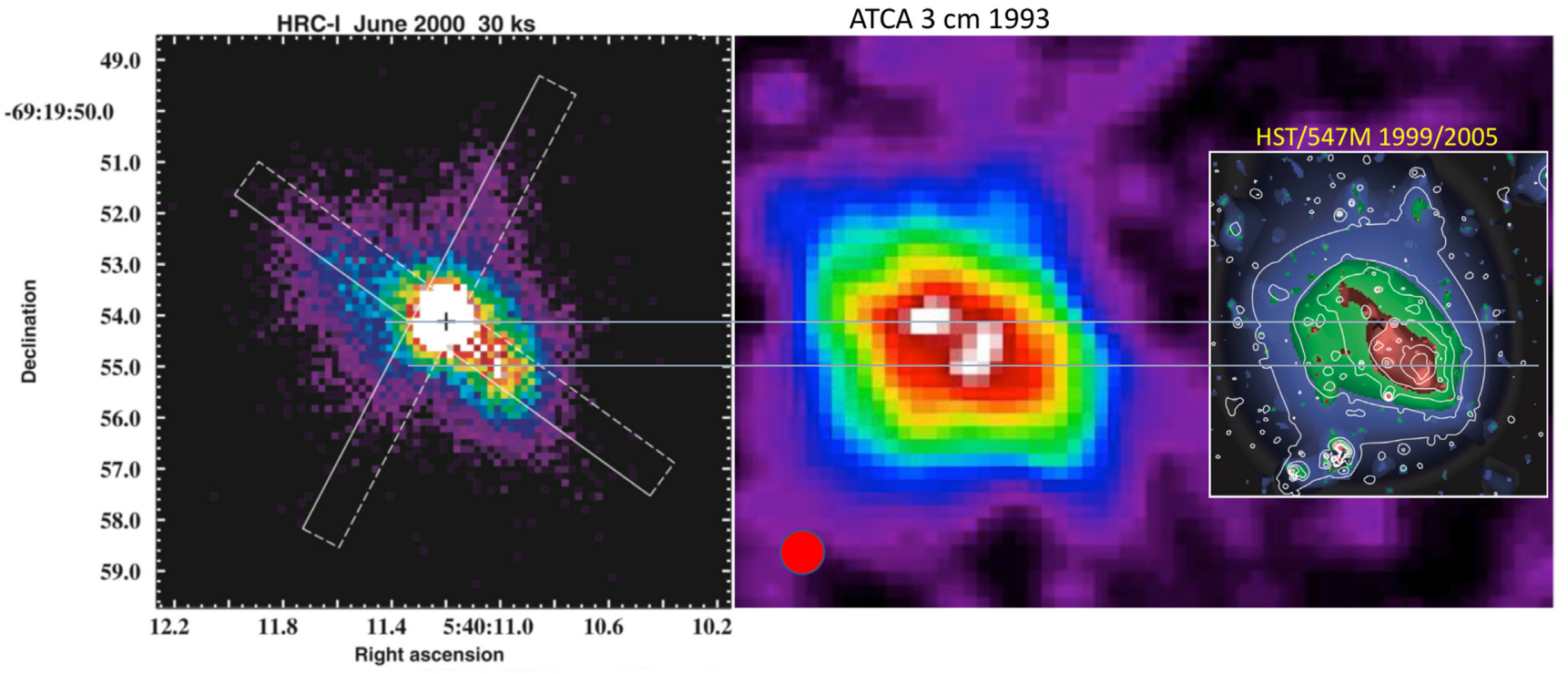

Figure 3. Zoom in of the $3 \mathrm{~cm}$ map (middle panel), showing two regions of strong emission (white areas), namely the pulsar region, and the 'blob' $\sim 1$ ". 5 south-west of the pulsar. This panel is similar to that in Dickel et al. (2002), but brings out structures more clearly. The filled red circle is the 0." 8 HPBW. The left-hand panel shows a Chandra 11 arcsec $\times 11$ arcsec X-ray map obtained with the High-Resolution Camera (HRC-I) in 1999. Again, the two dominating emission centres are seen. This panel, and the rightmost panel, showing the optical continuum as observed with HST/F547M, are from Lundqvist et al. (2011). Note that the HST colour map is for the 2005 epoch, and that contours are for 1999. All images are to the same scale, and the grey horizontal lines have been drawn through the pulsar position, and another through the blob at its 1999 position in the optical. In the optical image, data were wavelet filtered, after the pulsar and stars had been subtracted. The position of the pulsar in this panel is marked by a black cross. See the text for further details.

2005. If the blob is a multiwavelength feature/entity, it does not come as a surprise that the blob in the X-ray image from 2000 lines up well with the optical image from the same year, and that the radio image from 1995 shows a blob emission centred slightly closer to the pulsar than on images from 1999/2000. From Fig. 3 this seems to be the case. However, the overall structure of the PWN is similar at all wavelengths, although the relative flux densities within the PWN vary with frequency.

\subsection{Fluxes in the ACA and AKARI bands}

The peak flux density of SNR 0540-69.3 in Band 4 is $30.97 \mathrm{mJy}$ beam $^{-1}$ and the rms $0.291 \mathrm{mJy}^{\text {beam }}{ }^{-1}$. For Band 6, the corresponding numbers are 19.33 and $0.348 \mathrm{mJy}^{-1}$ beam $^{-1}$, respectively. These and other image properties are given in Table 2 . While the $\mathrm{S} / \mathrm{N}$ of PWN0540 in Band 4 is $\approx 106$ after our improved cleaning and self-calibration (see Section 2.2), the $\mathrm{S} / \mathrm{N}$ of the brightest pixel in the SNR shell features is $\sim 18$, so it is clear that the factor of 


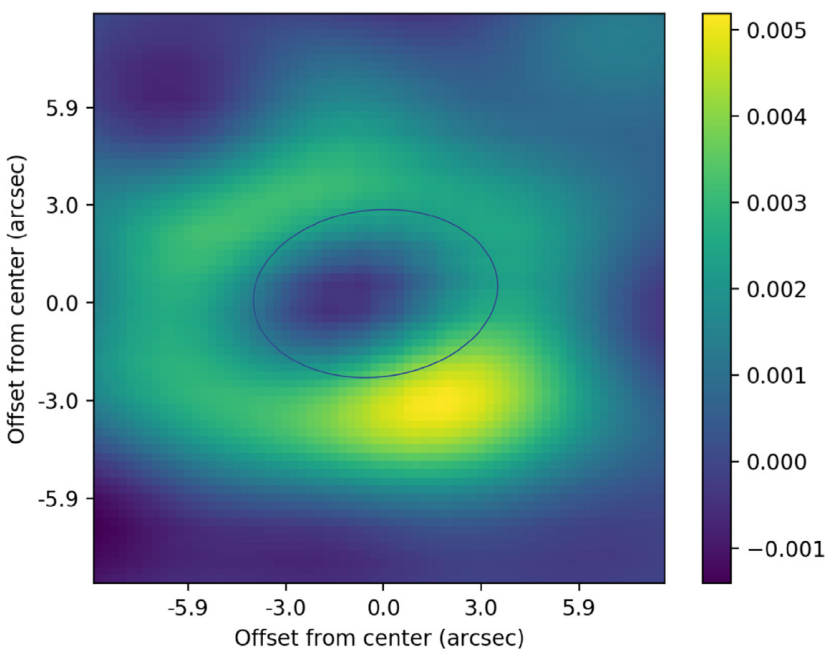

Figure 4. Difference in flux density (in Jy beam ${ }^{-1}$ ) between ACA Band 6 data and a point source smeared with the $7^{\prime \prime} .5 \times 5^{\prime \prime} .2$ beam (shown as an oval; see also Fig. 1). The modelled point source had the same surface peak flux as in the ACA image, and was placed at the position of the peak flux density in the ACA image. The yellow/green ring-like structure is excess flux due to the extended structure of the PWN, showing that the Band 6 data are partially spatially resolved. Note the particularly strong flux density in the direction of the blob to the south-west (cf. Fig. 3).

$\sim 2$ improvement in $\mathrm{S} / \mathrm{N}$ provided by self-calibration results in a significantly more robust detection of this extended feature (and we note that these SNR shell features were not detected in the initial shallow-cleaned images; the deeper cleaning and self-calibration described in Section 2.2 were essential for the detection of these features). Regarding uncertainties, as stated in Table 2, there is an uncertainty in the absolute flux calibration, which is estimated to be 5 per cent for Band 4 and 10 per cent for Band 6, as detailed in the ALMA Proposer's Guide ${ }^{4}$ (section A.9.2) and the ALMA Technical Handbook $^{5}$ (chapter 10). The flux uncertainty quoted in Table 2 is a combination of this and the rms.

The integrated flux density from the PWN would be the same as the peak flux density if the PWN were unresolved. This is not the case, since PWN0540 is partially resolved in both ACA bands. To test this we made an experiment were we assume that the source is a point source, so that the modelled spatial flux density distribution essentially becomes the beam distribution. We then normalized the peak flux density of this image to the observed image for each band. The difference between the observed image and the model is shown in Fig. 4 for Band 6. The net flux density is at zero level in the centre, as expected, but positive residuals are clearly displayed outside the HPBW oval, especially in the direction towards the blob.

We can use the result from this experiment to estimate a correction factor for the integrated flux density from the PWN, compared to the observed peak flux densities. This correction factor is simply the net flux density, as displayed in Fig. 4 for Band 6, relative to that measured from a point source. We obtain the correction factors $\sim 1.19$ and $\sim 1.50$ for Bands 4 and 6 , respectively. The correction factor and its estimated uncertainty are included in Table 4 for Band 6 . The situation is more complicated for Band 4 , since for this band

\footnotetext{
${ }^{4}$ https://almascience.eso.org/documents-and-tools/cycle7/alma-proposersguide

${ }^{5} \mathrm{https}$ ://almascience.eso.org/documents-and-tools/latest/documents-and- $\mathrm{t}$ ools/cycle7/alma-technical-handbook
}

Table 4. Observed multiwavelength integrated flux densities of PWN0540. ${ }^{\mathrm{a}}$

\begin{tabular}{|c|c|c|c|}
\hline $\begin{array}{l}\text { Frequency } \\
\mathrm{GHz}\end{array}$ & Instrument & $\begin{array}{c}\text { Flux density } \\
\text { mJy }\end{array}$ & Source $^{b}$ \\
\hline $1.513 \pm 0.064$ & ATCA $20 \mathrm{~cm}$ & $68 \pm 7^{c}$ & 1 \\
\hline $2.290 \pm 0.064$ & ATCA $13 \mathrm{~cm}$ & $62 \pm 6^{c}$ & 1 \\
\hline $4.790 \pm 0.064$ & ATCA $6 \mathrm{~cm}$ & $54 \pm 5^{c}$ & 1 \\
\hline $5.824 \pm 0.064$ & ATCA $6 \mathrm{~cm}$ & $60 \pm 6^{c}$ & 1 \\
\hline $8.640 \pm 0.064$ & ATCA $3 \mathrm{~cm}$ & $51 \pm 5^{c}$ & 1 \\
\hline $145.0 \pm 7.9$ & ACA Band 4 & $30.5 \pm 1.6^{c, d}$ & 2 \\
\hline $223.5 \pm 9.9$ & ACA Band 6 & $29.1 \pm 3.0^{c}$ & 2 \\
\hline $4350 \pm 110$ & Spitzer MIPS Ch2 & $<366$ & 3 \\
\hline$(1.26 \pm 0.21) \times 10^{4}$ & Spitzer MIPS Ch1 & $13.19 \pm 3.95$ & 3 \\
\hline$(1.30 \pm 0.15) \times 10^{4}$ & AKARI MIR-L L24 & $17.9 \pm 1.2$ & 2 \\
\hline$(1.92 \pm 0.36) \times 10^{4}$ & AKARI MIR-L L15 & $6.29 \pm 1.02^{\mathrm{c}}$ & 2 \\
\hline$(2.84 \pm 0.54) \times 10^{4}$ & AKARI MIR-S S11 & $4.62 \pm 0.27^{c}$ & 2 \\
\hline$(3.94 \pm 0.73) \times 10^{4}$ & Spitzer IRAC Ch4 & $5.10 \pm 0.74^{c}$ & 3 \\
\hline$(4.16 \pm 0.50) \times 10^{4}$ & AKARI MIR-S S7 & $3.38 \pm 0.24^{c}$ & 2 \\
\hline$(5.31 \pm 0.66) \times 10^{4}$ & Spitzer IRAC Ch3 & $3.61 \pm 0.46^{c}$ & 3 \\
\hline$(6.76 \pm 0.76) \times 10^{4}$ & Spitzer IRAC Ch2 & $2.19 \pm 0.27^{c}$ & 3 \\
\hline$(8.54 \pm 0.90) \times 10^{4}$ & Spitzer IRAC Ch1 & $1.77 \pm 0.23^{c}$ & 3 \\
\hline$(9.55 \pm 1.70) \times 10^{4}$ & AKARI NIR N3 & $2.26 \pm 0.20^{c}$ & 2 \\
\hline$(1.41 \pm 0.12) \times 10^{5}$ & $\mathrm{VLT} / \mathrm{NACO} / K_{s}$ & $0.801 \pm 0.035^{\mathrm{c}}$ & 4 \\
\hline$(1.83 \pm 0.19) \times 10^{5}$ & VLT/NACO/H & $0.675 \pm 0.027^{c}$ & 4 \\
\hline$(2.36 \pm 0.20) \times 10^{5}$ & $\mathrm{VLT} / \mathrm{NACO} / J$ & $0.541 \pm 0.022^{c}$ & 4 \\
\hline$(3.68 \pm 0.57) \times 10^{5}$ & $H S T / \mathrm{F} 814 \mathrm{~W}^{\mathrm{e}}$ & $0.481 \pm 0.014$ & 4 \\
\hline$(3.76 \pm 0.43) \times 10^{5}$ & $H S T / F 791 \mathrm{~W}$ & $0.437 \pm 0.009^{c}$ & 5 \\
\hline$(3.76 \pm 0.43) \times 10^{5}$ & HST/F791W & $0.426 \pm 0.013^{c}$ & 4 \\
\hline$(4.48 \pm 0.43) \times 10^{5}$ & $H S T / \mathrm{F} 675 \mathrm{~W}^{f}$ & $0.472 \pm 0.018$ & 4 \\
\hline$(5.50 \pm 0.36) \times 10^{5}$ & $H S T / \mathrm{F} 547 \mathrm{M}$ & $0.267 \pm 0.005^{c}$ & 5 \\
\hline$(5.71 \pm 0.86) \times 10^{5}$ & $H S T / \mathrm{F}^{2} 55 \mathrm{~W}^{g}$ & $0.346 \pm 0.017$ & 4 \\
\hline$(6.89 \pm 1.13) \times 10^{5}$ & $H S T / \mathrm{F} 450 \mathrm{~W}^{h}$ & $0.361 \pm 0.022$ & 4 \\
\hline$(9.00 \pm 0.64) \times 10^{5}$ & HST/F336W & $0.124 \pm 0.002$ & 5 \\
\hline$(9.00 \pm 0.64) \times 10^{5}$ & $H S T / F 336 \mathrm{~W}$ & $0.211 \pm 0.022^{c}$ & 4 \\
\hline
\end{tabular}

Notes. ${ }^{a}$ Near-infrared observations, and observations at higher frequencies, are dereddened integrated flux densities, according to their sources.

${ }^{b}$ References: (1) Brantseg et al. (2014), (2) this paper, (3) Williams et al. (2008),

(4) Mignani et al. (2012), and (5) Serafimovich et al. (2004).

${ }^{c}$ Included in the power-law fits in Figs 6 and 7.

${ }^{d}$ Includes correction for extended emission from the SNR shell.

${ }^{e}$ Includes [S III] $\lambda \lambda 9069,9532$.

${ }^{f}$ Includes [N II] $\lambda \lambda 6548,6583, \mathrm{H} \alpha$, and [S II] $\lambda \lambda 6716,6731$.

${ }^{g}$ Includes [O III] $\lambda \lambda 4959,5007$.

${ }^{h}$ Includes [O II] $\lambda \lambda 3726,3729$ and [O III] $\lambda \lambda 4959,5007$.

the correction factor is affected by flux from the SNR shell being smeared into the PWN region.

For Band 4, we have therefore also made another test that relies on an assumption that the underlying structures of the PWN and the SNR shell are similar at $3 \mathrm{~cm}$ and in the ACA bands. This assumption is best checked for Band 6 image with its higher spatial resolution, by smearing the $3 \mathrm{~cm}$ image with the ACA Band 6 beam, and then subtract this from the observed ACA Band 6 image. The residuals are found to be consistent with noise, which means that the intrinsic structures are similar at $3 \mathrm{~cm}$ and in Band 6 (and therefore also in Band 4), at least at the levels of signal-to-noise and spatial resolution of the ACA bands.

This intrinsic similarity between radio and millimetre structures allows us to improve on the correction for the integrated flux density of the PWN in Band 4. We did this by first constructing two images. For the first image, we only considered the PWN area of the $3 \mathrm{~cm}$ map, and smeared this with the ACA Band 4 beam. We call this Image $_{4 \mathrm{PWN}}$. We also made a simulated image of the SNR shell, Image $_{4 \text { shell }}$, where we first masked out the PWN from the $3 \mathrm{~cm}$ map, and then smeared the rest with the ACA Band 4 beam. We show this process in Fig. 5. We then removed the simulated shell image Image $_{4 \text { shell }}$ from the observed Band 4 image, Image ${ }_{4 \mathrm{obs}}$, thereby 

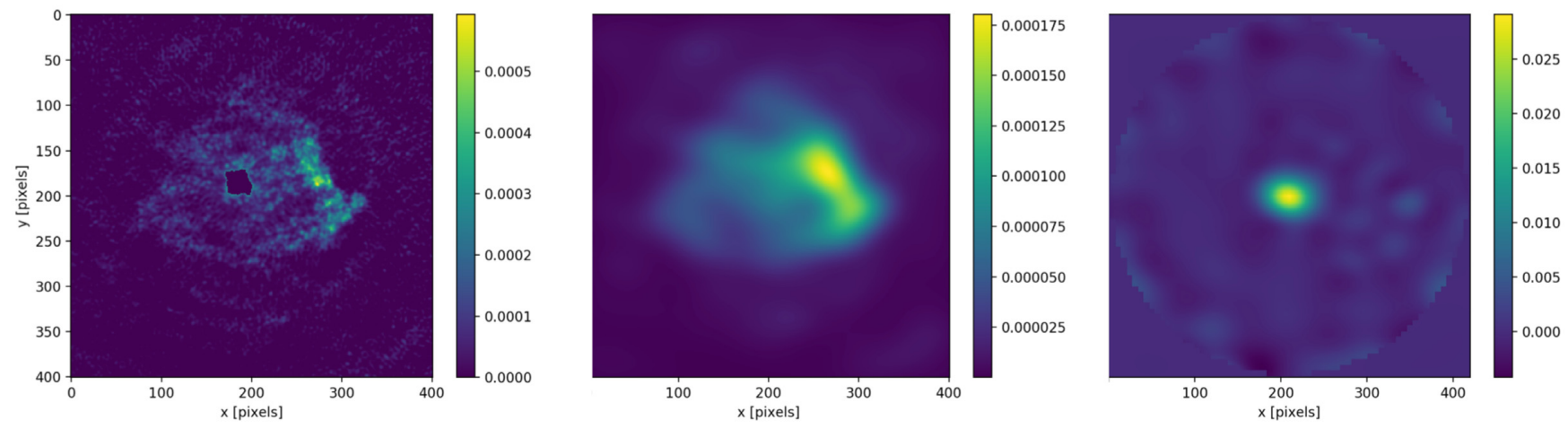

Figure 5. $3 \mathrm{~cm}$ image of SNR 0540-69.3, with the PWN masked out (left-hand panel). To obtain the middle panel, we smeared the $3 \mathrm{~cm}$ image with the ACA Band 4 beam. This simulates the structure of the pure remnant shell in Band 4, and is named Image 4 shell in Section 3.2. The image in the panel to the right is

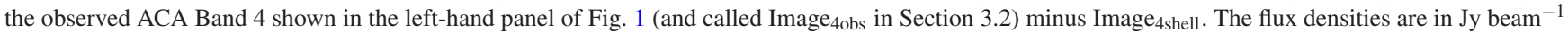

creating the right-hand panel of Fig. 5. This last step can be written as

Image $_{4 \text { diff }}=$ Image $_{4 \mathrm{obs}}-B \times$ Image $_{4 \mathrm{SNR}}$.

We then created a null image by removing Image $_{4 \mathrm{PWN}}$ from Image $_{4 \text { diff }}$, i.e.

Image $_{4 \text { resid }}=$ Image $_{4 \text { diff }}-A \times$ Image $_{4 \mathrm{PWN}}$.

The difference was tuned through the constants $A$ and $B$ to achieve zero net flux density at the positions of the PWN and SNR, respectively. The ratio $A / B$ can be used to estimate a power-law index for the spectral range between $3 \mathrm{~cm}$ and ACA Band 4 for the SNR shell emission, if the index is known for the PWN.

$\alpha_{\nu, \mathrm{SNR}}=\alpha_{\nu, \mathrm{PWN}}+0.816 \lg (A / B)$.

In our analysis, we arrive at $A / B=3.8 \pm 0.2$, so that $\alpha_{\nu \text {, SNR }}-$ $\alpha_{\nu, \mathrm{PWN}}=0.47 \pm 0.03$. Brantseg et al. (2014) estimated $\alpha_{\nu, \mathrm{SNR}}-$ $\alpha_{v, \text { PWN }}=0.50 \pm 0.04$ for radio alone. Our result is fully consistent with the same difference in power-law index continuing into the millimetre regime.

The peak flux density of the image in the right-hand panel of Fig. 5, i.e. Image ${ }_{4 \text { diff }}$, is $29.07 \mathrm{mJy}_{\text {beam }}{ }^{-1}$, which is $\sim 6$ per cent lower than that before the correction for emission from the remnant shell (cf. Table 2). Finally, we used Image ${ }_{4 \text { diff }}$ to calculate the flux correction factor for the PWN, as we did for Band 6, to arrive at the value 1.05 , which we include in the integrated flux density for Band 4 in Table 4 . This correction factor is smaller than the value we obtained before compensating for the SNR shell, as expected.

For the AKARI data, we used 343.34, 74.956, 38.258, 16.034, and $8.0459 \mathrm{Jy}$ as fluxes for zero magnitude for the five IRC bands N3, S7 S11, L15, and L24, respectively (Tanabé et al. 2008; Kato et al. 2012). The AKARI flux densities are listed in Table 4, along with the frequency interval for which the normalized filter transparency is $\geq 50$ per cent.

\section{DISCUSSION}

We have used integrated flux densities in Table 4 to fit power laws to different parts of the synchrotron spectrum. Not all data in the table have been included in these fits, as will be detailed below. Concentrating first on the radio/mm-region, we have used the ACA flux densities in Section 3.2 together with radio flux densities from Brantseg et al. (2014). We note that there is some inconsistency in Brantseg et al. (2014) with regard to the $6 \mathrm{~cm}$ flux densities at 4.790 and $5.824 \mathrm{GHz}$ in their figs 6 and 11, compared to their table 2. We have assumed that the flux densities in their table 2 are correct (since they agree with their fig. 11), and have used the flux densities uncertainties shown in their fig. 6 (as no uncertainties are provided in their table 2). With this caveat in mind, we estimate $\alpha_{v}=0.17 \pm 0.02$ for the frequency interval 1.449-233.50 GHz. This is shown in Fig. 6

The uncertainty of $\alpha_{\nu, \text { radio/mm }}$ and its $1 \sigma$ interval were estimated using the same method as in Serafimovich et al. (2004), i.e. utilizing a Monte Carlo approach to construct 10000 power-laws to fit 10000 simulated sets of data, where the flux uncertainty is assumed to have a Gaussian distribution, and the frequency distribution to be evenly distributed within the frequency bins given in Table 4 . We have then ordered the 10000 simulated data sets in order of fitted $\alpha_{v \text {, radio/mm }}$ values, and from this assigned the median value to be the preferred

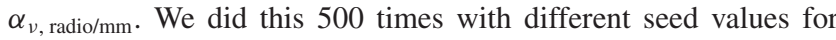
the randomizer, and then took the average value for the power-law index to be the final estimate of the index. The $1 \sigma$ boundaries for $\alpha_{\nu \text {, radio/mm }}$ shown in Fig. 6 come from the constraint that 68 per cent of the constructed power laws must lie within the $1 \sigma$ boundaries.

To make a similar fit to the IR/UV part of the spectrum, we included the Spitzer IRAC IR data from Williams et al. (2008), the AKARI data in Table 4, and the VLT/NACO data of Mignani et al. (2012). The HST data for PWN0540 in the optical/UV have been published by Serafimovich et al. (2004) and Mignani et al. (2012). In Serafimovich et al. (2004), care was taken to only consider HST filters that do not include strong nebular spectral lines. On the contrary, most of the filters considered by Mignani et al. (2012) include the strongest lines in the nebula (cf. Kirshner et al. 1989; Serafimovich et al. 2005; Morse et al. 2006), as we have indicated in Table 4. To check the contribution from spectral lines to the flux in HST filters, we have chosen to study the influence by [O III] $\lambda \lambda 4959,5007$ on the flux in F555W. Fluxes for various lines from the whole PWN were estimated by Williams et al. (2008). For [O III $]$ 25007, they used the line flux deduced by Morse et al. (2006), and assumed that the size of the PWN, as seen in [O III], is a factor of $\approx 4$ larger than the area covered by the slit used by Morse et al. (2006). This means that the dereddened line flux from the whole PWN is $f_{[\mathrm{O} \mathrm{II}] \lambda 5007} \approx 2.7 \times 10^{-13} \mathrm{erg} \mathrm{cm}^{-2} \mathrm{~s}^{-1}$. However, the slit used by Morse et al. (2006) covers the brightest areas in [O III] (cf. Sandin et al. 2013), so a correction factor of 4 is too large. We have taken a more conservative approach for the flux of [O III] $\lambda \lambda 4959,5007$ than Williams et al. (2008), and adopt $f_{[\mathrm{O} \mathrm{II}] \lambda 5007}=2.0 \times 10^{-13} \mathrm{erg} \mathrm{cm}^{-2} \mathrm{~s}^{-1}$ and $f_{[\mathrm{O} \mathrm{III]}] \lambda 4959}$ to be 


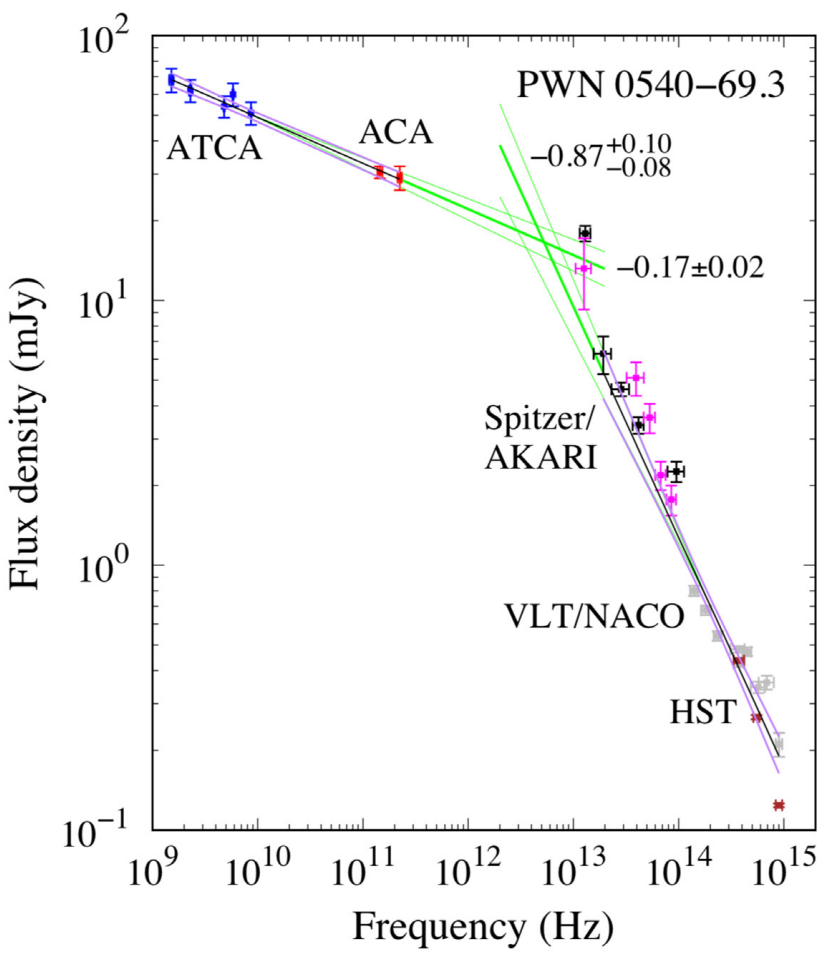

Figure 6. Multiwavelength spectrum of PWN0540. Our estimated flux densities in ACA Bands 4 and 6 are shown by red symbols, and the radio flux densities estimated by Brantseg et al. (2014) are marked in blue. The best power-law fit to those data has a spectral index of $\alpha_{\nu, \mathrm{mm}}=0.17 \pm 0.02$. (The figure gives spectral slopes, i.e. $-\alpha_{\nu}$.) A similar fit to the UV/IR part gives $\alpha_{\nu, \mathrm{UVIR}}=0.87_{-0.10}^{+0.08} .1 \sigma$ intervals for the power laws are drawn in purple. The data included in this fit are the Spitzer IRAC data of Williams et al. (2008) (in magenta), the AKARI data reported here (in black), and the VLT/NACO data of Mignani et al. (2012) (in grey). Data for the UV/optical regime published by Serafimovich et al. (2004) (in brown) and by Mignani et al. (2012) (in grey) are all shown in the figure, but as detailed in the text, not all those data were included in the power-law fit. Extrapolations of the two power laws (in green), intersect at $v \sim 5.2 \times 10^{12} \mathrm{~Hz}$. Extrapolations of the $1 \sigma$ intervals are also drawn in green.

one third of that, in accordance with the 3:1 ratio of the transition probabilities of the two lines (e.g. Osterbrock \& Ferland 2006). To compare with the continuum emission, we use the flux in the F547M filter (cf. Table 4) and the continuum spectral slope $F_{v} \propto v^{-0.87}$ (see below). With the filter transmission considered, we arrive at an [O III] $\lambda \lambda 4959,5007$ flux that is $\sim 60$ percent of the continuum flux within F555W, i.e. the integrated continuum flux density at $5.71 \times 10^{14} \mathrm{~Hz}$ is probably closer to $0.20-0.25 \mathrm{mJy}$ than the $\sim 0.35$ mJy listed in Table 4. Had we used the [O III] $\lambda 5007$ line flux estimated by Williams et al. (2008), the derived integrated continuum flux density in F555W would have been $\lesssim 0.20 \mathrm{mJy}$. Based on this, we have discarded HST filters that encapsulate strong nebular lines from our analysis.

The HST filters we have included are F336W, F547M, and F791W. For F791W, the fluxes estimated by Serafimovich et al. (2004) and Mignani et al. (2012), using the same data from 1999 October 17, agree to within 3 per cent, and we used an average value of those. This contrasts the situation for F336W where the two teams obtained very different results. Mignani et al. (2010, 2012) argue that this is not mainly because the groups analyse different data sets (from 1999 October 17 and 2007 June 21), but that the so-called charge transfer efficiency effect, which is important for F336W, is treated better by them. We have therefore chosen to only include the Mignani et al. (2012) measurement for that filter. F547M does not include any strong nebular lines (Serafimovich et al. 2004), and this band was included in our power-law estimate. All data used in our power-law fits are highlighted in Table 4 by the asterisk marked ' $c$ '. With this in mind, we arrive at $\alpha_{\nu, \mathrm{UVIR}}=0.87_{-0.10}^{+0.08}$ for the IR/UV part of the synchrotron spectrum. [Had we included the F336W flux of Serafimovich et al. (2004), the power-law index would only have changed to $0.91_{-0.10}^{+0.08}$. This is less steep than $\alpha_{v}=1.48_{-0.08}^{+0.09}$ by Serafimovich et al. (2004) and $1.27 \pm 0.3$ by Brantseg et al. (2014), but steeper than $\alpha_{v}=0.56 \pm 0.03$ by Mignani et al. (2012). Our results supersede all those estimates. The $-0.87_{-0.08}^{+0.10}$ spectral slope for the IR/UV range is drawn and marked in Fig. 6. Note the flux excess for the discarded $H S T$ filters compared to the power-law fit, due to line emission contamination.

In the IR, the integrated AKARI flux densities in the range (2.34.7) $\times 10^{13} \mathrm{~Hz}$ are lower than those measured by Spitzer, despite the fact that there is line contribution mainly from to [S IV] $10.5 \mu \mathrm{m}$ (Williams et al. 2008; see also Fig. 8). This underlines difficulties in background subtraction in the IR. In general, the AKARI data line up better with the full IR/UV power law, except for the N3 and L24 bands. The L24 band is not part of the power-law fits in Fig. 6 though.

In Fig. 6, an extrapolation of the high-energy power law undershoots the flux level of the $\sim 24 \mu \mathrm{m}$ AKARI data by several $\sigma$. This could signal a contribution to the emission from dust with $T_{\text {dust }} \sim$ 40-65 K, as argued for by Williams et al. (2008). However, broadline emission in [C IV] $25.9 \mu \mathrm{m}$ and [Fe II] $26.0 \mu \mathrm{m}$ contributes to the integrated AKARI flux density in the AKARI L24 band. Using the line fluxes estimated by Williams et al. (2008), and taking into account the transparency in the L24 band at $26 \mu \mathrm{m},{ }^{6}$ we estimate that lines can contribute up to $\sim 5-6$ per cent of the integrated AKARI flux density in the L24 band, which only marginally decreases the need for dust to fit the $24 \mu \mathrm{m}$ data (see below).

Extrapolations of the low-frequency and the IR/UV power laws in Fig. 6 intersect at $v_{\text {break }} \sim 5.2 \times 10^{12} \mathrm{~Hz}$. If we use the $1 \sigma$ limits in Fig. 6, we obtain $v_{\text {break }}=\left(5.2_{-2.8}^{+3.6}\right) \times 10^{12} \mathrm{~Hz}$. If one assumes that this break is due to synchrotron cooling of relativistic electrons, one can estimate the magnetic field strength, $B$, where the electrons are being injected. Equalling the lifetime of synchrotron-emitting electrons, $\tau_{\text {synch }}=6 \times 10^{11} B^{-3 / 2} v_{\text {break }}^{-1 / 2} \mathrm{~s}$ (Pacholczyk 1970; Brantseg et al. 2014), to the age of the remnant, assumed to be $1100 \mathrm{yr}$ (Reynolds 1985), this translates into $B=\left(3.9_{-0.7}^{+1.1}\right) \times 10^{-4} \mathrm{G}$. This is higher than the findings of Manchester et al. (1993b) and Brantseg et al. (2014), who both estimated $B \sim 2.5 \times 10^{-4} \mathrm{G}$, but somewhat lower than a recent value derived from $\mathrm{X}$-rays, $B=\left(7.8_{-2.8}^{+45}\right) \times 10^{-4} \mathrm{G}(\mathrm{Ge}$ et al. 2019).

The spectral break between the low- and high-frequency parts of the synchrotron spectrum gives a steepening in power-law index by $\Delta=0.70_{-0.10}^{+0.08}$, which is slightly larger than the expected standard change of 0.5 due to synchrotron losses in a homogeneous medium. Although $\Delta=0.5$ is not fully excluded from our spectral fits, Reynolds (2009) discusses possibilities with $\Delta>0.5$, and how this could signal inhomogeneities in regions where the relativistic electrons are injected. There is also the possibility that the $\Delta \sim$ 0.7 break in power-law index is not due to synchrotron losses, but signals two populations of relativistic electrons, as in the model of Bucciantini et al. (2011, cf. Introduction).

\footnotetext{
${ }^{6}$ http://svo2.cab.inta-csic.es/svo/theory/fps3/index.php?mode = browse
} 


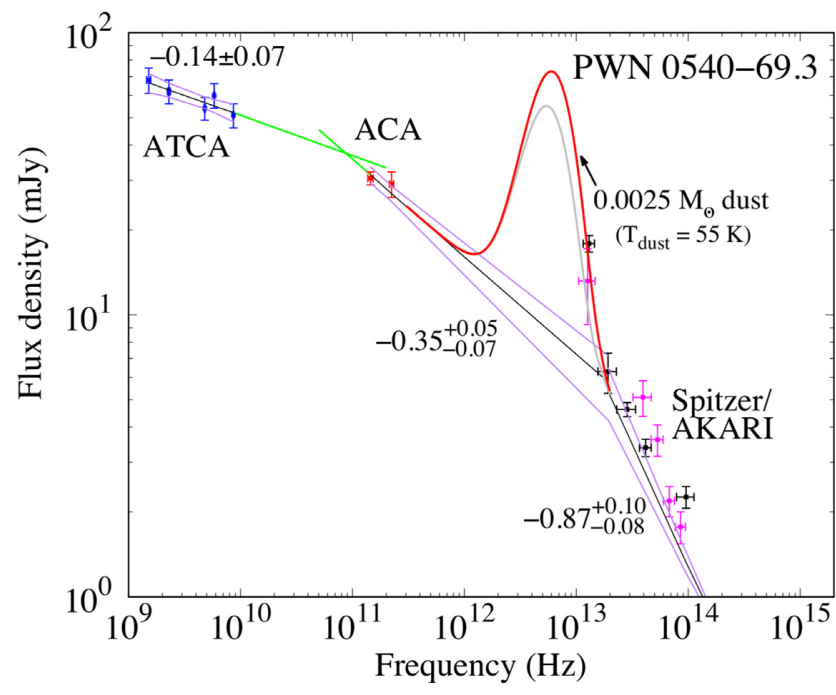

Figure 7. Same as Fig. 6, but only showing the ATCA, ACA, Spitzer, and AKARI data. One separate power-law fit has been made for the ATCA data, and another for the ACA data plus the AKARI $15 \mu \mathrm{m}$ data. Spectral indices for the various frequency intervals are shown $(\alpha$, radio $=0.14 \pm 0.07$ and $\alpha_{v, \mathrm{~mm} / \mathrm{IR}}=0.35_{-0.05}^{+0.07}$ ). (The figure gives spectral slopes, i.e. $-\alpha_{v}$.) Extrapolations of the radio-IR power laws intersect at $\sim 8.4 \times 10^{10} \mathrm{~Hz}$, which can be compared with a similar break at $\sim 3 \times 10^{10} \mathrm{~Hz}$ break for the Crab Nebula. Emission from $2.5 \times 10^{-3} \mathrm{M}_{\odot}$ of silicate dust with a temperature of $T_{\text {dust }}=55 \mathrm{~K}$ is also included (in red), to model the AKARI and Spitzer data longward of $\sim 20 \mu \mathrm{m}$. For a comparison, we also include a model (in grey) with preferred values by Williams et al. (2008), namely the dust mass $3 \times 10^{-3} \mathrm{M}_{\odot}$ and $T_{\text {dust }}=50 \mathrm{~K}$

Rather than a single power-law break at $v_{\text {break }} \sim 5.2 \times 10^{12} \mathrm{~Hz}$, a dual break is also possible. The Crab PWN may serve as a template. As described in the Introduction section, the Crab PWN has a spectral break at $\sim 3 \times 10^{10} \mathrm{~Hz}$, where the spectral index changes from $\alpha_{v} \approx 0.3$ in the radio to $\alpha_{v} \approx 0.42$ at higher frequencies (Gomez et al. 2012). There could be a similar break for PWN0540. If we fit a power law to the radio data alone, the spectral index becomes $\alpha_{\nu \text {, radio }}=0.14 \pm 0.07$. Similarly, we can also fit a power law to the ACA data and the AKARI L15 data at $(1.92 \pm 0.36) \times 10^{13} \mathrm{~Hz}$, and the power law for this range then is $\alpha_{v, \mathrm{~mm} / \mathrm{IR}}=0.35_{-0.05}^{+0.07}$. As shown in Fig. 7, the two power laws intersect at $\sim 8.4 \times 10^{10} \mathrm{~Hz}$, i.e. somewhat higher frequency than the $\sim 3 \times 10^{10} \mathrm{~Hz}$ break for the Crab. With dual power laws, the total steepening in power-law index becomes $\Delta=0.73_{-0.12}^{+0.11}$, which is again greater than $\Delta=0.5$ at $\sim 2 \sigma$ level.

However, the difference in power-law index between $\alpha_{v, \mathrm{~mm} / \mathrm{IR}}$ and $\alpha_{v, \text { UVIR }}$ is $0.52 \pm 0.11$, which is fully consistent with a cooling break of 0.5. Returning to the model of Bucciantini et al. (2011; see also Lyutikov et al. 2019), there could be two populations of relativistic electrons, one being responsible for radio emission and the other giving rise to the $\mathrm{mm} / \mathrm{IR}$ part. The latter could experience a break due to synchrotron losses, which is reflected in a power-law break of $\sim 0.5$ at $\nu_{\text {break }} \sim 2 \times 10^{13} \mathrm{~Hz}$. If we use $B=8 \times 10^{-4} \mathrm{G}$, as indicated from X-rays, $\tau_{\text {synch }} \sim 190 \mathrm{yr}$, which is markedly less than the age of the remnant, although not as low as $\tau_{\text {synch }} \sim 2.6 \mathrm{yr}$ estimated by Petre et al. (2007), from a presumed cooling break in X-rays instead of at $\sim 2 \times 10^{13} \mathrm{~Hz}$. Inspired by the model of Lyutikov et al. (2019), which suggests a cooling break at $\sim 0.01 \mathrm{eV}$ for the Crab PWN, $\tau_{\text {synch }} \sim 190 \mathrm{yr}$ seems more likely than $\sim 3 \mathrm{yr}$ for PWN0540.
A dual spectral break in the radio-IR range for the synchrotron emission in PWN0540 as shown in Fig. 7 allows for the possibility of dust emission to explain excess emission at $24 \mu \mathrm{m}$. Such a dust component was discussed by Williams et al. (2008), and in Fig. 7 we include emission (shown in red, and highlighted with a black arrow) from $2.5 \times 10^{-3} \mathrm{M}_{\odot}$ of silicate dust with a temperature of $T_{\text {dust }}=$ $55 \mathrm{~K}$ (red solid line). The dust emission was calculated assuming optically thin forsterite dust. The flux density $F_{\lambda}$ at wavelength $\lambda$ can be written as

$F_{\lambda}=\frac{\kappa_{\lambda} M_{\text {dust }} B_{\lambda}\left(T_{\text {dust }}\right)}{D^{2}}$,

where $M_{\text {dust }}$ is the dust mass, $D$ is the distance to PWN0540 (i.e. $50 \mathrm{kpc}), B_{\lambda}\left(T_{\text {dust }}\right)$ is the Planck function for temperature $T_{\text {dust }}$, and $\kappa_{\lambda} \propto \lambda^{-\beta}$ is the mass absorption coefficient of the selected type of dust (e.g. Hildebrand 1983; Matsuura 2017). The values of $\kappa_{\lambda}$ and $\beta$ are temperature dependent. We have assumed small-sized dust particles, and interpolated $\kappa_{\lambda}$ and $\beta$ in temperature using the results reported by Mennella et al. (1998). The choice of silicate dust is motivated by the fact that Nozawa et al. (2003) find that silicates may dominate in supernova ejecta of massive progenitors, especially in mixed ejecta. Williams et al. (2008) show that the presumed dust component increases in strength towards longer wavelengths up to the end of their spectra at $\sim 37 \mu \mathrm{m}$. This is consistent with the model in Fig. 7 where the curve in red (dust + synchrotron) peaks at $\sim 50 \mu \mathrm{m}$. Our dust mass estimate is consistent with the $\sim 3 \times 10^{-3} \mathrm{M}_{\odot}$ of silicate dust with $T_{\text {dust }}=50 \pm 8 \mathrm{~K}$ calculated by Williams et al. (2008). In Fig. 7, we include (in grey) a model with those parameters. As can be seen, this model does not fit the integrated AKARI L24 flux density well. This is not surprising since the model of Williams et al. (2008) was tuned to fit the Spitzer data. The inclusion of the AKARI L24 data pushes the derived dust temperature to a slightly higher value than that estimated by Williams et al. (2008).

As can be seen in Fig. 7, the AKARI and Spitzer flux densities are larger than the extrapolation along the best optical-IR synchrotron fit. This could indicate a dust contribution all the way up in frequency to the VLT/NACO bands, i.e. up to $\sim 1.4 \times 10^{14} \mathrm{~Hz}$. This would require dust temperatures ranging between $\sim 50$ and $60 \mathrm{~K}$, or lower, and up to several hundred Kelvins. Although warm dust is seen in SNe (cf. Matsuura 2017, and references therein), detected PWN-embedded dust usually has temperatures lower than $100 \mathrm{~K}$ (Chawner et al. 2019). SNR 1E 0102.2-7219 in the Small Magellanic Cloud may be an exception at first glance. Sandstrom et al. (2009) argue that small amounts $\left(\sim 2 \times 10^{-5} \mathrm{M}_{\odot}\right)$ of newly formed forsterite dust with $T_{\text {dust }} \approx 140 \mathrm{~K}$ are associated with the supernova ejecta, and a pulsar was recently reported for this remnant. However, no PWN (Vogt et al. 2018) has yet been found, so SNR 1E 0102.2-7219 is probably more similar to Cas A than PWN0540 when it comes to the central source and its immediate surroundings.

A more likely reason for the high AKARI and Spitzer flux densities $\lesssim 20 \mu \mathrm{m}$ is line contribution. For example, as shown in Fig. 8, the AKARI S11 band observations of PWN0540 (Williams et al. 2008 ) include [S IV] $10.5 \mu \mathrm{m}$ at $f_{[\mathrm{S} \mathrm{IV}]} \approx 7.3 \times 10^{-14} \mathrm{erg} \mathrm{cm}^{-2} \mathrm{~s}^{-1}$, and [Ne II] $12.8 \mu \mathrm{m}$ at $f_{[\mathrm{Ne} I \mathrm{II}} \approx 5.0 \times 10^{-14} \mathrm{erg} \mathrm{cm}^{-2} \mathrm{~s}^{-1}$. In Fig. 8 , the blue solid line is part of the $\alpha_{v}$, UVIR $=0.87$ power-law fit in Figs 6 and 7, and the fluxes of the spectral lines are the broad lines $\left(\mathrm{FWHM} \sim 1000 \mathrm{~km} \mathrm{~s}^{-1}\right.$ ) reported by Williams et al. (2008). Taking into account the transmission of the AKARI S11 filter (cf. Fig. 8), the measured flux in the spectral lines is $\sim 20$ per cent of the continuum flux. If we add this estimated line contribution to 


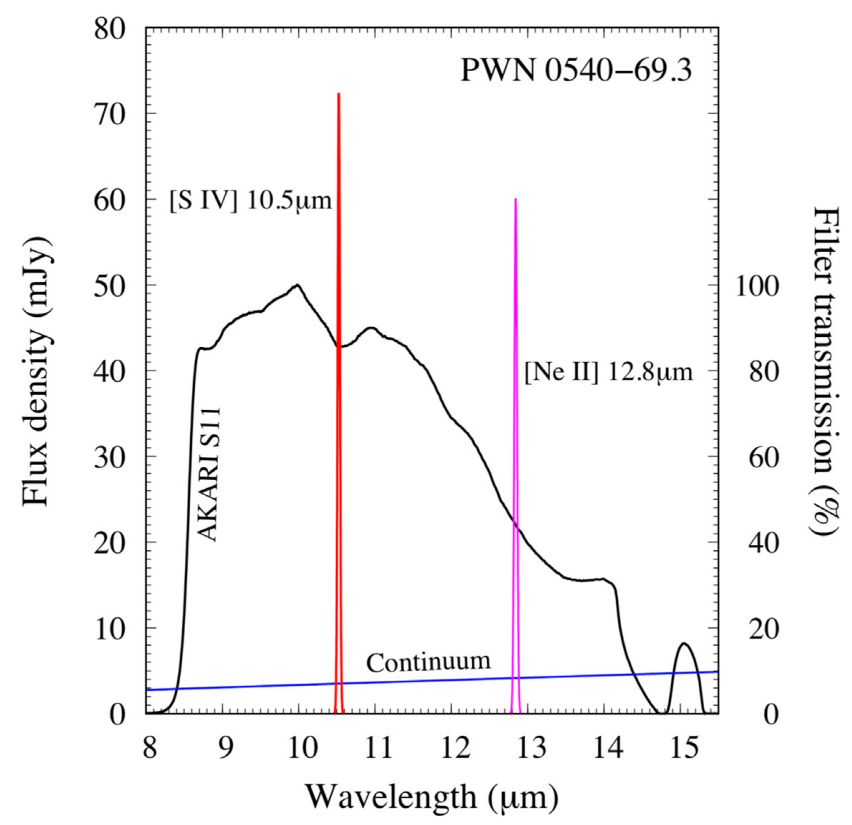

Figure 8. Emission from PWN0540 in the AKARI S11 band. The blue solid line is a power-law fit to the synchrotron continuum, adopting the $\alpha_{v}$, UVIR $=$ 0.87 power-law index from Figs 6 and 7. The two strongest spectral lines in this band, [S IV] $10.5 \mu \mathrm{m}$ and [Ne II] $12.8 \mu \mathrm{m}$, are also shown. Fluxes and widths of the lines are from Williams et al. (2008). The normalized filter transmission of the S11 band is highlighted by the black solid line. See the text for further details.

the power-law fit value for the continuum in the AKARI S11 band, which is $3.81 \mathrm{mJy}$ at $2.84 \times 10^{13} \mathrm{~Hz}$, the total flux density becomes $4.57 \mathrm{mJy}$. This agrees excellently with the observed integrated flux density in the AKARI S11 band that is $4.62 \pm 0.27 \mathrm{mJy}$ (cf. Table 4).

In our flux estimates of the continuum emission from PWN0540, we have made no correction for emission from PSR0540. Serafimovich et al. (2004) made a detailed discussion of this for the optical/UV part, and their results indicate that the pulsar would contribute $\sim 5$ percent if the pulsar were not spatially resolved. The multiwavelength compilation of Serafimovich et al. (2004) also shows that the PWN and the pulsar are of equal strength in X-rays (cf. Fig. 3), but that in radio the pulsar contribution is negligible compared to that from the PWN. This trend is confirmed by Mignani et al. (2012) whose results point to an $\sim 3$ percent pulsar contribution in the $H$ band. We can therefore safely ignore any pulsar contribution to the integrated flux densities in the ACA, AKARI, and Spitzer bands. As outlined before, uncertainties due to spectral lines are larger. Further observations of PWN0540 in the near- and mid-infrared, preferably spectroscopic to remove the flux from spectral lines, should solve whether dust contributes to the emission measured by Spitzer and AKARI. For example, the James Webb Space Telescope, with its expected spectral range between 0.6 and $28.5 \mu \mathrm{m}$ and $0 . " 1$ optical spatial resolution, is an ideal tool for this task.

In our analysis in Section 3.2, we obtained $\alpha_{\nu, \text { SNR }}-\alpha_{\nu, \text { PWN }}=$ $0.47 \pm 0.03$ for the difference in spectral index between the remnant shell and the PWN for the spectral interval 8.6-145 GHz. Using Table 4 , we find $\alpha_{v, \text { PWN }}=0.19 \pm 0.04$, and hence $\alpha_{v \text {, SNR }}=$ $0.64 \pm 0.05$. Brantseg et al. (2014) found $\alpha_{v, \mathrm{SNR}}=0.65 \pm 0.01$ for the range 1.4-8.6 GHz, fully consistent with a constant spectral slope over two orders of magnitude in frequency. Our value for the remnant shell is also consistent with typical values for synchrotron radiation from other radio SNRs (Green 2009). It is also consistent with the spectrum for the synchrotron emission from the circumstellar ring around SN 1987A where $\alpha_{v, 87 \mathrm{~A}}=0.70 \pm 0.06$ (Cigan et al. 2019) for the same extended frequency interval we have discussed for SNR 0540-69.3 (see also Zanardo et al. 2014).

\section{CONCLUSIONS}

We have observed SNR 0540-69.3 with the ACA in Bands 4 (137$153 \mathrm{GHz})$ and $6(213.5-233.5 \mathrm{GHz})$, which is a new frequency range for this object. As the HPBW of ACA is similar to the size of the remnants PWN in Band $6\left(7^{\prime \prime} .5 \times 5^{\prime \prime} .2\right)$, and slightly larger than the PWN in Band $4\left(10^{\prime \prime} .8 \times 8\right.$ "..3), the PWN was only partially resolved.

We also use deep radio observations obtained with the ATCA at $3 \mathrm{~cm}$, with an HPBW of $\approx 0.0^{\prime \prime}$. We used the 3 -cm data as a template for the emission in the millimetre range, and smeared these data with the HPBWs of ACA Bands 4 and 6. These simulations suggest a similar overall structure at millimetre wavelengths compared to that at $3 \mathrm{~cm}$. In particular, we recover the strong emission $\sim 1^{\prime \prime} .5$ to the south-west of the pulsar seen at other wavelengths. If we include published radio flux densities (Brantseg et al. 2014), we obtain a synchrotron spectrum $F_{v} \propto v^{-0.17 \pm 0.02}$ for the frequency interval 1.449-233.50 GHz.

To draw conclusions about how the radio-millimetre wavelength range of the spectrum joins to the synchrotron spectrum at higher frequencies, and whether there could be dust in the PWN, we have evaluated published data in the UV, optical, and IR, as well as included previously unpublished AKARI IR data. We show that some of these data are seriously contaminated by spectral line emission, and are therefore not suitable for analysing the continuum emission. For the UV/IR part of the synchrotron spectrum, we find $F_{v} \propto v^{-0.87_{-0.08}^{+0.10}}$. The break between the radio-millimetre and UV/IR power law occurs at $v_{\text {break }}=\left(5.2_{-2.7}^{+3.6}\right) \times 10^{12} \mathrm{~Hz}$, which can be used to estimate the magnetic field strength, $B=\left(3.9_{-0.7}^{+1.1}\right) \times 10^{-4} \mathrm{G}$, in the PWN, if the lifetime of synchrotron-emitting electrons is as large as the remnant age. This field strength is slightly higher than previous findings of Manchester et al. (1993b) and Brantseg et al. (2014).

To explain the high observed flux from the PWN at $24 \mu \mathrm{m}$, and at the same time allow for a break in the synchrotron spectrum between radio and millimetre wavelengths as is seen in the $\mathrm{Crab}$, we find that dust with a temperature of $\sim 50-60 \mathrm{~K}$ is needed. For $55 \mathrm{~K}$, the mass is $\sim 2.5 \times 10^{-3} \mathrm{M}_{\odot}$ for forsterite dust. The inferred break between radio and millimetre wavelengths would occur at $\sim 8 \times 10^{10} \mathrm{~Hz}$, with the spectral slope changing from $-0.14 \pm 0.07$ in the radio to $-0.35_{-0.07}^{+0.05}$ in the millimetre-to-far-IR range. The total change in power-law index based between radio and the optical/UV is $\Delta \sim$ 0.7 , with $\Delta=0.5$, as in the standard case of synchrotron losses in a homogeneous medium, being excluded at $\sim 2 \sigma$ level, regardless of whether there is a spectral break at $\sim 8 \times 10^{10} \mathrm{~Hz}$ or not.

However, there is a possible scenario for PWN0540, which is inspired by a recent model by Lyutikov et al. (2019) for the Crab PWN and which can accommodate a cooling break of $\sim 0.5$. In this model, our spectra reveal two populations of synchrotronemitting relativistic electrons: one that emits in the radio with $F_{v} \propto v^{-0.14 \pm 0.07}$ and another that emits in the millimetre-to-UV range. In the millimetre-to-far-IR range, the latter component emits according to $F_{v} \propto v^{-0.35_{-0.07}^{+0.05}}$. Although shallower than $\propto v^{-0.5}$, uncertainties are large enough for this component to be consistent with Fermi acceleration. The observed steepening in power-law index by $0.52 \pm 0.11$ to $F_{v} \propto v^{-0.87_{-0.08}^{+0.10}}$ for $v \gtrsim 2 \times 10^{13} \mathrm{~Hz}$ could 
be due to synchrotron cooling, as in the Crab. For a magnetic field strength of $B \approx 8 \times 10^{-4} \mathrm{G}$, as indicated by recent $\mathrm{X}$-ray studies (Ge et al. 2019), the synchrotron cooling time is $\sim 190 \mathrm{yr}$.

For ACA Band 4, we clearly detect the SNR shell of SNR 054069.3, and we can constrain the spectrum of its synchrotron emission. We find that its spectral slope is steeper than that for the PWN. The spectral slope is $-0.64 \pm 0.05$ between 8.6 and $145 \mathrm{GHz}$, which agrees with previous estimates for radio alone, as well as standard values for other remnants, and the radio/millimetre spectrum of the ring of SN 1987A.

\section{ACKNOWLEDGEMENTS}

We thank the anonymous referee for important comments. PL acknowledges support from the Swedish Research Council. The work of YS was partially supported by the RFBR grant 1629-13009. This paper makes use of the following ALMA data: ADS/JAO.ALMA\#2017.1.01391.S. ALMA is a partnership of ESO (representing its member states), NSF (USA), and NINS (Japan), together with NRC (Canada), MOST and ASIAA (Taiwan), and KASI (Republic of Korea), in cooperation with the Republic of Chile. The Joint ALMA Observatory is operated by ESO, AUI/NRAO, and NAOJ. The National Radio Astronomy Observatory (NRAO) is a facility of the National Science Foundation operated under cooperative agreement by Associated Universities, Inc. The Australia Telescope Compact Array (ATCA) is part of the Australia Telescope National Facility that is funded by the Australian Government for operation as a National Facility managed by CSIRO. The ATCA data reported here were obtained under Program C014. This research has also made use of the NASA Astrophysics Data System (ADS) Bibliographic Services. Furthermore, this research has made use of the SVO Filter Profile Service (http://svo2.cab.inta-csic.es/theory/fps/) supported from the Spanish MINECO through grant AYA201784089. MM acknowledges support from STFC Ernest Rutherford fellowship (ST/L003597/1).

\section{REFERENCES}

Brantseg T., McEntaffer R. L., Bozzetto L. M., Filipovic M., Grieves N., 2014, ApJ, 780, 50

Bucciantini N., Arons J., Amato E., 2011, MNRAS, 410, 381

Bykov A., Gehrels N., Krawczynski H., Lemoine M., Pelletier G., Pohl M., 2012, Space Sci. Rev., 173, 309

Caraveo P. A., Bignami G. F., Mereghetti S., Mombelli M., 1992, ApJ, 395, L103

Cerutti B., Uzdensky D. A., Begelman M. C., 2012, ApJ, 746, 148

Chawner H. et al., 2019, MNRAS, 483, 70

Chevalier R. A., 2006, astro-ph/0607422

Cigan P. et al., 2019, ApJ, 51, 27

Clausen-Brown E., Lyutikov M., 2012, MNRAS, 426, 1374

De Looze I., Barlow M. J., Swinyard B. M., Rho J., Gomez H. L., Matsuura M., Wesson R., 2017, MNRAS, 465, 3309

De Luca A., Mignani R. P., Caraveo P. A., Bignami G. F., 2007, ApJ, 667, 77

Dickel J. R. et al., 2002, in Slane P. O., Gaensler B., eds, ASP Conf. Ser. Vol. 271, Neutron Stars in Supernova Remnants. Astron. Soc. Pac., San Francisco, p. 195

Dwek E., Arendt R. G., 2015, ApJ, 810, 75

Ge M. Y. et al., 2019, Nat. Astron., 3, 1122

Gelfand J. D., Slane P. O., Zhang W., 2009, ApJ, 703, 2051

Gomez H. L. et al., 2012, ApJ, 760, 96

Gotthelf E. V., Wang Q. D., 2000, ApJ, 532, L117

Green D. A., 2009, Bull. Astron. Soc. India, 37, 45

Hildebrand R. H., 1983, Q. J. R. Astron. Soc., 24, 267

Indebetouw R. et al., 2014, ApJ, 782, L2

Kato D. et al., 2012, AJ, 144, 179

Kirshner R. P., Morse J. A., Winkler P. F., Blair W. P., 1989, ApJ, 342, 260

MNRAS 496, 1834-1844 (2020)
Lundqvist N. et al., 2011, MNRAS, 413, 611

Lyutikov M., Temim T., Komissarov S., Slane P., Sironi L., Comisso L., 2019, MNRAS, 489, 2403

Manchester R. N., Mar D. P., Lyne A. G., Kaspi V. M., Johnston S., 1993a, ApJ, 403, 29

Manchester R. N., Staveley-Smith L., Kesteven M. J., 1993b, ApJ, 411, 756

Martin J., Torres D. F., Rea N., 2012, MNRAS, 427, 415

Matsuura M. et al., 2011, Science, 333, 1258

Matsuura M. et al., 2015, ApJ, 800, 50

Matsuura M., 2017, in Alsabati A. M., Murdin P., eds, Handbook of Supernovae, Springer Int. Publ. AG, Cham, p. 2125

McMullin J. P., Waters B., Schiebel D., Young W., Golap K., 2007, in Shaw R. A., Hill F., Bell D. J., eds, ASP Conf. Ser. Vol. 376, Astronomical Data Analysis Software and Systems XVI. Astron. Soc. Pac., San Francisco, p. 127

Mennella V., Brucato J. R., Colangelli L., Palumbo P., Rotundi A., Bussoletti E., 1998, ApJ, 496, 1058

Middleditch R. N., Pennypacker C. R., 1985, Nature, 313, 659

Mignani R. P., Sartori A., De Luca A., Rudak B., Słowikowska A., Kanbach G., Caraveo P. A., 2010, A\&A, 515, 110

Mignani R. P., De Luca A., Hummel W., Zajczyk A., Rudak B., Kanbach G., Słowikowska A., 2012, A\&A, 544, 100

Mignani R. P. et al., 2019, ApJ, 871, 246

Morse J. A., Smith N., Blair W. P., Kirshner R. P., Winkler P. F., Hughes J. P., 2006, ApJ, 644, 188

Nozawa T., Kozasa T., Umeda H., Maeda K., Nomoto N., 2003, ApJ, 598, 785

Onaka T. et al., 2007, PASJ, 59, S401

Osterbrock D. E., Ferland G. J., 2006, Astrophysics of Gaseous Nebulae and Active Galactic Nuclei. Univ. Sci. Books, Sausalito, CA, USA

Owen P. J., Barlow M. J., 2015, ApJ, 801, 141

Pacholczyk A. G., 1970, Radio Astrophysics. Nonthermal Processes in Galactic and Extragalactic Sources. Freeman, San Francisco, CA, USA

Park S., Hughes J. P., Slane P. O., Mori K., Burrows D. N., 2010, ApJ, 710, 948

Petre R., Hwang U., Holt S. S., Safi-Harb S., Williams R. M., 2007, ApJ, 662,997

Porth O., Vorster M. J., Lyutikov M., Engellbrecht N. E., 2016, MNRAS, 460, 4135

Reynolds S. P., 1985, ApJ, 291, 152

Reynolds S. P., 2009, ApJ, 703, 1

Reynolds S. P., Pavlov G. G., Kargaltsev O., Klingler N., Renaud M., Mereghetti S., 2017, Space Sci. Rev., 207, 175

Rho J. et al., 2018, MNRAS, 479, 510

Sandin C., Lundqvist P., Lundqvist N., Björnsson C.-I., Olofsson G., Shibanov Yu. A., 2013, MNRAS, 435, 329

Sandstrom K. M., Bolatto A. D., Stanimirovic S., van Loon J. T., Smith J. D., 2009, ApJ, 696, 2138

Sault R. J., Teuben P. J., Wright M. C. H., 1995, in Shaw R., Payne H. E., Hayes J. J. E., eds, ASP Conf. Ser. Vol. 77, Astronomical Data Analysis Software and Systems IV. Astron. Soc. Pac., San Francisco, p. 433

Serafimovich N. I., Shibanov Yu. A., Lundqvist P., Sollerman J., 2004, A\&A, 425,1041

Serafimovich N. I., Lundqvist P., Shibanov Yu. A., Sollerman J., 2005, Adv. Space Res., 35, 1106

Seward F. D., Harnden F. R., Jr, Helfand D. J., 1984, ApJ, 287, L19

Sironi L., Spitkovsky A., 2011, ApJ, 741, 39

Tanabé T. et al., 2008, PASJ, 60, S375

Temim T., Dwek E., Arendt R. G., Borkowski K. J., Reynolds S. P., Slane P., Gelfand J. D., Raymond J. C., 2017, ApJ, 836, 129

Vogt F. P. A., Bartlett E. S., Seitenzahl I. R., Michael A., Ghavamian P., Ruiter A. J., Terry J. P., 2018, Nat. Astron., 2, 465

Williams B. J. et al., 2008, ApJ, 687, 1054

Zanardo G. et al., 2014, ApJ, 796, 82

Zharikov S. V., Zyuzin D. A., Shibanov Y. A., Mennickent R. E., 2014, A\&A, 554, A120

This paper has been typeset from a $\mathrm{T}_{\mathrm{E}} \mathrm{X} / \mathrm{LT} \mathrm{E} \mathrm{X}$ file prepared by the author. 